\title{
Midstream Clean-Catch Urine Culture Obtained by Stimulation Technique versus Catheter Specimen Urine Culture for Urinary Tract Infections in Newborns: A Paired Comparison of Urine Collection Methods
}

\author{
Nilgun Altuntas ${ }^{\mathrm{a}}$ Basak Alan $^{\mathrm{b}}$ \\ ${ }^{a}$ Division of Neonatology, Department of Pediatrics, Ankara Yıldırım Beyazıt University Medical Faculty, Ankara,

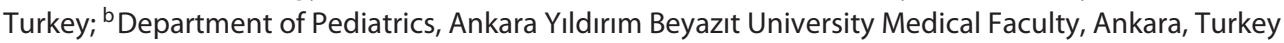

\section{Highlights of the Study}

- Midstream clean-catch urine (MS-CCU) collection can be done safely, quickly, and effectively in the newborns using bladder stimulation and paravertebral lumbar massage.

- MS-CCU can be used as an alternative to other noninvasive urine collection methods.

- Because of its high contamination rate, it cannot replace the catheter for the diagnosis of urinary tract infections.

\section{Keywords}

Midstream clean-catch urine $\cdot$ Catheter specimen urine . Newborn · Urinary tract infection

\footnotetext{
Abstract

Objective: The group of Herreros Fernández developed a new, safe, quick, and successful technique for collecting midstream clean-catch urine (MS-CCU) in newborns based on bladder stimulation and lumbar paravertebral massage maneuvers. The purpose of this study was to compare the contamination rates of catheter specimen urine (CSU) and MS-CCU by a lumbar/sacral stimulation technique in newborns. Materials and Methods: Full-term newborns ranging in age from 2 to 28 days who needed an investigation for a presumed urinary tract infection (UTI) were included in the study. Two samples, MS-CCU by lumbar/sacral stimulation
}

technique and CSU, were collected consecutively for each patient. Suitable samples were obtained from 90 patients. Results: The contamination rate in MS-CCU cultures ( $n=$ 24/90, 26.66\%) was higher than in CSU cultures ( $n=9 / 90$, $10 \%$ ), and the difference was statistically significant ( $p=$ 0.039). Thirteen patients had UTI according to both samples (14.14\%). In urine analysis, while there was no statistically significant difference in bacteriuria $(p=0.61)$ and nitrite positivity ( $p=0.14$ ) between patients with and without UTI, pyuria $(p=0.01)$ and leukocyte esterase positivity $(p=0.01)$ were higher in patients with UTI, and the difference was statistically significant. Conclusion: The contamination rate in MS-CCU cultures was two and a half times greater than in the CSU culture samples. Thus, MS-CCU cannot replace the catheter for the diagnosis of UTI.

(C) 2019 The Author(s) Published by S. Karger AG, Basel

\begin{tabular}{ll}
\hline KARGER & $\begin{array}{l}\text { (c) } 2019 \text { The Author(s) Karger } \\
\text { Published by S. Karger AG, Basel }\end{array}$ \\
karger@karger.com & $\begin{array}{l}\text { This is an Open Access article licensed under the Creative Commons } \\
\text { Attribution-NonCommercial-4.0 International License (CC BY-NC) } \\
\text { (http://www.karger.com/Services/OpenAccessLicense), applicable to } \\
\text { the online version of the article only. Usage and distribution for com- } \\
\text { mercial purposes requires written permission. }\end{array}$
\end{tabular}




\section{Introduction}

Because of nonspecific clinical signs of urinary tract infection (UTI) and suboptimal sensitivity (75-85\%) [16] of the urinalysis (UA) for the diagnosis of UTI, urine culture is considered to be the gold standard tool in the diagnosis of UTI in children. Nevertheless, occasionally inappropriate urine samples cause contamination and confusion over results, and repeating the same procedure leads to loss of time and delay of appropriate treatment. Therefore, the method of urine collection used should have a minimum risk of contamination. The American Academy of Pediatrics (AAP) UTI guideline, developed for babies over 2 months, suggests that the diagnosis of UTI should require an abnormal UA (pyuria and/or bacteriuria) in addition to a positive urine culture (at least 5,0000 colony-forming units [cfu] per milliliter of a uropathogen cultured from suprapubic aspiration [SPA] and catheter specimens) [5]. However, there is no guide demonstrating the safety of urine analysis for neonates and which urine collection method should be used.

SPA and catheter specimens have the least contamination rates; however, these methods are painful for the baby, stressful for the practitioner, and require an experienced team. Therefore, usually for nonseptic children, noninvasive methods of urine collection are preferred. The bagged urine collection method is simple and noninvasive but has a high contamination rate and a high false positive rate of 85-99\% [7]; hence, it is not suitable for use as a diagnostic tool for UTI. Therefore, noninvasive and reliable methods for precontinent children continue to be investigated. The National Institute for Health and Clinical Excellence recommend that urine be obtained by the clean-catch urine (CCU) technique from all young children in cases of suspected UTI. It is the preferred method for urine collection because CCU is noninvasive, requires less technical expertise, and has a lower contamination rate than urine collection bags [8]. Some studies demonstrate that CCU collection could be considered as a reliable noninvasive technique when compared to the gold standard SPA $[9,10]$. Nevertheless, there are reports stating that the parents find the CCU time-consuming and messy [7]. Therefore, novel methods to obtain more rapid CCU in precontinent children were examined in previous studies.

Herreros Fernández et al. [11] developed a new, safe, quick, and successful technique based on bladder stimulation and lumbar paravertebral massage maneuvers to collect midstream clean-catch urine (MS-CCU) in newborns. In a previous study, we have shown that this meth- od is effective in neonates [12]. We also examined the contamination rate in a previous study but did not compare MS-CCU with a gold standard method.

The purpose of the present study was to compare the contamination rates of catheter specimen urine (CSU) and MS-CCU cultures in newborns. Our secondary aim was to investigate whether UA, white blood cell (WBC), $\mathrm{C}$-reactive protein (CRP), and fever were significant for UTI in newborns. To our knowledge, this is the first study to compare the CSU and MS-CCU obtained by bladder stimulation and lumbar paravertebral massage maneuvers in newborn.

\section{Materials and Methods}

This prospective study was carried out in a tertiary Neonatal Intensive Care Unit from September 2016 to September 2018. After the local ethics committee approved the study, informed consent was obtained from the legal guardian of each child. Full-term newborns ranging in age from 2 to 28 days who needed an investigation for a presumed UTI were assessed for eligibility to participate in the study. Neonates who were fully fed orally were included in the study. Exclusion criteria from the study were nutritional problems, oliguria or anuria, serious illnesses affecting the mobility of the baby, antibiotic treatment or UTI during the previous 30 days, congenital urinary tract abnormalities, and infection of the external genitalia. Urine samples obtained using the MSCCU by the lumbar/sacral stimulation method and using the CSU method, were collected consecutively for each patient $3 \mathrm{~h}$ apart.

Firstly, bladder stimulation and lumbar paravertebral massage maneuvers were applied to the babies as described by Herreros Fernández et al. [11]. When the bladder was full again after the next feeding, urine was obtained using a catheter from the same babies. After the perineum was cleaned with povidone iodine, a 5 -Fr feeding tube was inserted into the urethra for the catheterization. None of the male infants in our study were circumcised. The foreskin was gently retracted before catheterization in patients whose foreskin retraction status was classified as complete until urethral meatus was seen. In both methods (clean catch and catheterization), the first few drops were routinely discarded to prevent contamination from the distal urethra. Urine samples were sent to the laboratory immediately in sterile containers for prompt UA and culture.

Analysis of uncentrifuged urine was done in the BT Uricell-2 analyzer, and data (WBC count, bacteriuria, nitrites, and leukocyte esterase [LE] positivity) were obtained for each patient. Pyuria was defined as a WBC count of $\geq 10 / \mathrm{mm}^{3}$ [5]. Any bacterium in the urine was considered as positive bacteriuria because to the best of our knowledge there is no quantitative recommendation for bacteriuria.

Growth of a single pathogenic organism at a concentration of $\geq 10,000 \mathrm{cfu} / \mathrm{mL}$ was defined as positive urine culture. UTI was diagnosed as growth of a single and same pathogenic organism at a concentration of $\geq 10,000 \mathrm{cfu} / \mathrm{mL}$ in both urine samples. Urine culture was considered to be contaminated if it had mixed bacterial growth, growth of even one nonpathogenic bacterium (Lactobacil- 
Table 1. Demographic characteristics of the study population

\begin{tabular}{lc}
\hline Gestational week & $38(37-41)$ \\
Birth weight, g & $3,304.66 \pm 452.95$ \\
Gender & $54(40)$ \\
$\quad$ Male & $36(60)$ \\
$\quad$ Female & $7(2-28)$ \\
Postnatal age, days & $13(14.14)$ \\
UTI & \\
\hline
\end{tabular}

Data are presented as medians (minimum-maximum), mean \pm standard deviation, or $n(\%)$. UTI, urinary tract infection. lus species, coagulase-negative Staphylococcus and Corynebacterium species), had colony counts $<10,000 \mathrm{cfu} / \mathrm{mL}$ or any bacterial growth while urine culture result is negative by the other method. Sterile samples (i.e., negative cultures) were identified as undeveloped. Urine cultures of infants with inadequate reproduction in both urine cultures $\left(<10^{4} \mathrm{cfu} / \mathrm{mL}\right)$ were repeated by taking another urine sample using a catheter. The babies were monitored for complications during the procedure. Blood culture, WBC, and CRP were analyzed in all patients.

\section{Statistical Analysis}

The normality of data was analyzed using the KolmogorovSmirnov test. The normally distributed variables were expressed as means (standard deviations), and the nonnormally distributed variables were expressed as medians (minimum-maximum). The $\chi^{2}$ test was used for categorical variables and expressed as observation counts (in percentages). Differences in the rates of contamination for MS-CCU and CSU were calculated using the $\chi^{2}$ test or Fisher's exact test where appropriate. The Mann-Whitney U test was used to compare of numerical variables distributed nonnormally. A two-sided $p$ value $<0.05$ was considered as statistically significant. We calculated the sensitivity and specificity of UA results (LE test and pyuria) for the prediction of UTIs. All analyses were performed with a commercially available software program (Statistical Package for the Social Sciences, version 16; SPSS Inc., Chicago, IL, USA).

\section{Results}

One hundred and ten neonates hospitalized for investigating fever of unknown origin $(n=5)$, hyperbilirubinemia $(n=50)$, vomiting $(n=7)$, respiratory distress $(n=$ 13 ), and those who required UA for various reasons ( $n=$ 35) between September 2016 and October 2018 were included in this study. Ten neonates were excluded due to dehydration $(n=5)$ or low oral intake $(n=5)$. Urine could not be obtained by massage from 10 babies. The success rate in collecting urine samples by massage was $90 \%$. The median time (IQR) for sample collection by massage was
Table 2. Contamination rates of CSU and MS-CCU cultures $(n=$ 90) according to gender

\begin{tabular}{lccl}
\hline & CSU & MS-CCU & $p$ value \\
\hline Contamination & $9(10)$ & $24(26.7)$ & 0.039 \\
$\quad$ Girls & $3(8.33)$ & $8(22.22)$ & 0.33 \\
$\quad$ Boys & $6(11.11)$ & $16(29.62)$ & 0.007 \\
$p$ value & 0.66 & 0.43 & \\
\hline
\end{tabular}

Data are presented as $n(\%)$. MS-CCU, midstream clean catch urine; CSU, catheter-specimen urine. $p$ value by $\chi^{2}$ test with Yates correction or Fisher's exact test where appropriate.

39 s. Urine samples were successfully collected from 90 babies by using the two methods. Demographic characteristics of babies are given in Table 1 .

Thirteen patients were diagnosed with UTI. Both urine cultures of these patients were positive. Ten patients in the MS-CCU group and 3 patients in the CSU group had false positive culture. The culture results of MS-CCU and CSU were consistent in 61 patients. The contamination rate was $10 \%$ in CSU samples and $26.66 \%$ in MS-CCU samples when the cutoff value for positive urine culture was defined as $\geq 10^{4} \mathrm{cfu} / \mathrm{mL}$ and the difference was statistically significant. There was no significant difference in urine contamination rates between girls and boys in both CSU and MS-CCU samples (Table 2).

There was no significant difference between the patients with or without UTI in terms of WBC count, CRP count, and fever.

In UA, while there was no statistically significant difference in bacteriuria and nitrite positivity between patients with and without UTI, pyuria and LE positivity were higher in patients with UTI, and the difference was statistically significant (Table 3). On the other hand, pyuria and LE positivity had low sensitivity and high specificity in terms of UTI, respectively.

\section{Discussion}

Bacterial contamination during urine collection makes diagnosis difficult in non-toilet-trained children. Recommended collection methods for small children are SPA and urethral catheterization [5]. Contamination rates for SPA are slightly lower than those for urethral catheterization [13], but it requires a more advanced skill set and has lower parental acceptance rate. Moreover, it is considered by most providers to be more invasive than catheterization 
Table 3. Characteristics of patients with $(n=13)$ and without UTI $(n=77)$

\begin{tabular}{lccc}
\hline & Patients with UTI & Patients without UTI & $p$ value \\
\hline WBC, cells/ $\mu \mathrm{L}$ & $9,000(7,500-17,000)$ & $10,000(5,400-29,200)$ & 0.96 \\
Fever+ & $2(15.38)$ & $11(14.28)$ & 0.59 \\
CRP, mg/dL & $0.16(0.01-0.62)$ & $0.14(0-4.12)$ & 0.89 \\
\hline Urinalysis & & & 0.14 \\
$\quad$ Nitrite+ & $1(7.69)$ & $0(0)$ & 0.01 \\
$\quad$ Pyuria & $5(38.46)$ & $8(10.38)$ & 0.61 \\
$\quad$ Bacteriuria+ & $2(15.38)$ & $7(9.1)$ & 0.01 \\
$\quad$ LE+ & $4(30.76)$ & $4(5.19)$ & \\
\hline
\end{tabular}

Data are presented as medians (minimum-maximum) or $n(\%)$. UTI, urinary tract infections; WBC, white blood cells; CRP, C-reactive protein; LE, leukocyte esterase; pyuria, a urine WBC count of $\geq 10 / \mathrm{mm}^{3}$. $p$ value by $\chi^{2}$ test with Yates correction or Fisher's exact test where appropriate.

and is not preferred. After Herreros Fernández et al. [11] developed a new, safe, quick, and successful technique based on bladder stimulation and lumbar paravertebral massage maneuvers for collecting MS-CCU in newborns, MS-CCU became an alternative to noninvasive urine collection methods in newborns. In our previous study, we showed that this new technique for obtaining urine samples was effective in newborns [12]. In the same study, we found the rate of contamination to be $24 \%$ in the experimental group when the cutoff value was defined as $10^{5} \mathrm{cfu} /$ $\mathrm{mL}$, and $18.4 \%$ when the cutoff value was defined as $10^{4}$ $\mathrm{cfu} / \mathrm{mL}$, but we did not compare this method with an invasive one. In the present study, we compared the contamination rates of CSU culture and MS-CCU culture in newborns. We used $\geq 10^{4} \mathrm{cfu} / \mathrm{mL}$ as the cutoff value and found that the contamination rate was $10 \%$ in CSU cultures and $26.7 \%$ in MS-CCU cultures. Studies show that the rates of contamination in the CCU vary between 4.5 and $51 \%$. While our catheter contamination rate was consistent with the literature $[8,13-15]$, our MS-CCU contamination rate was consistent with some studies $[8,16,17]$, but higher than the results of other studies $[9,10,14,18-22]$. We suggest that these variations were caused by differences in study design, study population, and different cutoff values. Other studies were performed in the pediatric age group with small infants. A recent study by Herreros et al. [14] was similar to our study design, but the study population and cutoff value were different from our study. They compared MS-CCU obtained by a technique based on bladder stimulation and lumbar paravertebral massage maneuvers and CSU in 60 patients less than 3 months of age (70\% were boys); surprisingly the CCU contamination rate (5\%) was lower than in CSU (8\%) [14]. While the catheter results were consistent with our results, the MS-CCU samples were much lower than ours. The difference is not likely to be due to the gender difference in the populations, because MS-CCU contamination rates of boys in our study group are higher than in girls, although the difference is not statistically significant. It is likely that the difference in age of the study populations is important. Furthermore, in the study by Herreros et al. [14], urine cultures were considered negative if $<1,000 \mathrm{cfu} / \mathrm{mL}$ in samples using catheterization or $<10,000 \mathrm{cfu} / \mathrm{mL}$ in samples using the clean-catch technique [14]. However, in our study, sterile or negative cultures were defined as those with no growth. Any growth with colony counts less than $10,000 \mathrm{cfu} / \mathrm{mL}$ or any bacterial growth when the urine culture result is negative by the other method was considered contamination. Thus, some urine cultures that we considered as contaminated were actually negative according to their criteria. The rate in the present study was slightly higher than that in our previous study (26.7 vs. $18.4 \%)$. We believe that this is mainly due to the difference in study design. In the current study, each child enrolled for the examination had two samples of urine collected consecutively using the CSU and MS-CCU methods. Sixteen patients in the CSU samples and 23 patients in the MS-CCU samples had positive culture with a growth rate of $\geq 10^{4} \mathrm{cfu} / \mathrm{mL}$. The same bacteria in both cultures grew $\left(\geq 10^{4} \mathrm{cfu} / \mathrm{mL}\right)$ in only 13 patients, and these patients were diagnosed with UTI. As a result, 10 patients with the MS-CCU samples and 3 patients with the CSU samples had false positive cultures. The contamination rate may have decreased if urine samples were not obtained with both methods for each patient, but the frequency of false UTI diagnosis could have increased. The rate of agreement between two urine collection methods was $74.44 \%$. 
We have been using MS-CCU obtained by bladder stimulation and lumbar paravertebral massage maneuvers as an alternative to sterile bag urine samples for selective catheterization in newborns with suspected UTI. In our previous study, we showed that this new technique in obtaining urine samples was safe, quick, and effective in newborns [12]. However, in order to make unequivocal conclusions, well-designed studies comparing these two noninvasive methods in terms of time, contamination rate, and success are needed.

Our secondary aim in the present study was to investigate whether UA, WBC, CRP, fever, and renal USG were significant for UTI in newborns. Thirteen patients (14.14\%) were diagnosed with UTI. In accordance with the literature, laboratory values such as WBC count, and CRP were not significantly different among infants with and without UTI $[2,23]$.

Enhanced UA is a rapid screening test for the presumptive diagnosis of UTI, but it cannot be a substitute for urine culture and should be used in conjunction with urine culture. While negative UA does not exclude UTI, positive UA does not indicate a definitive diagnosis of UTI. Several studies showed that UA has a good specificity and sensitivity in predicting UTI $[15,24]$. A systematic review has shown that nitrites and LE have good sensitivity and specificity in detecting UTI in older children but are less reliable in infants [25]. We found that there was no statistically significant difference between patients with and without UTI in terms of nitrite positivity and bacteriuria, whereas pyuria and LE positivity were statistically higher in patients with UTI. Pyuria and LE positivity had low sensitivity, but high specificity for UTI detection.

UTI in newborns is frequently complicated by bacteremia. In a retrospective study, $12.4 \%$ of the blood cultures in newborns who presented with UTI were positive [26]. However, urosepsis was significantly less frequent in community-acquired UTI than in nosocomial UTI. In our study all UTIs were community-acquired, and no positive blood culture was observed in any patient. In terms of fever, there was no difference between infants with and without UTI.

\section{Conclusion}

Given the high contamination rate, our results suggest that MS-CCU cannot replace the catheter but can be used as an alternative to other noninvasive urine collection methods for selective catheterization in newborns with suspected UTI. We also find that LE positivity and/or pyuria may be more meaningful in diagnosing UTI.

\section{Statement of Ethics}

The authors have no ethical conflicts to disclose.

\section{Disclosure Statement}

The authors have no conflicts of interest to disclose.

\section{Funding Sources}

No funding was secured for this study.

\section{Author Contributions}

Dr. Altuntas conceptualized and designed the study, acquired and interpreted the data, drafted the manuscript, and approved the final manuscript. Dr. Alan acquired the data and approved the final manuscript as submitted.

\section{References}

1 Bachur R, Harper MB. Reliability of the urinalysis for predicting urinary tract infections in young febrile children. Arch Pediatr Adolesc Med. 2001 Jan;155(1):60-5.

2 Bonadio W, Maida G. Urinary tract infection in outpatient febrile infants younger than 30 days of age: a 10 -year evaluation. Pediatr Infect Dis J. 2014 Apr;33(4):342-4.

3 Hoberman A, Wald ER. Urinary tract infections in young febrile children. Pediatr Infect Dis J. 1997 Jan;16(1):11-7.

4 Newman TB, Bernzweig JA, Takayama JI, Finch SA, Wasserman RC, Pantell RH. Urine testing and urinary tract infections in febrile infants seen in office settings: the Pediatric Research in Office Settings' Febrile Infant Study. Arch Pediatr Adolesc Med. 2002 Jan;156(1):44-54.

5 Roberts KB; Subcommittee on Urinary Tract Infection, Steering Committee on Quality Improvement and Management. Urinary tract infection: clinical practice guideline for the diagnosis and management of the initial UTI in febrile infants and children 2 to 24 months. Pediatrics. 2011 Sep;128(3):595610.

6 Shaw KN, McGowan KL, Gorelick MH, Schwartz JS. Screening for urinary tract infection in infants in the emergency department: which test is best? Pediatrics. 1998 Jun; 101(6):E1. Available from: www.pediatrics. org/cgi/content/full/101/6/E1

7 Liaw LC, Nayar DM, Pedler SJ, Coulthard MG. Home collection of urine for culture from infants by three methods: survey of parents' preferences and bacterial contamination rates. BMJ. 2000 May;320(7245):1312-3.

8 Tosif S, Baker A, Oakley E, Donath S, Babl FE. Contamination rates of different urine collection methods for the diagnosis of urinary tract infections in young children: an observational cohort study. J Paediatr Child Health. 2012 Aug;48(8):659-64. 
9 Amir J, Ginzburg M, Straussberg R, Varsano I. The reliability of midstream urine culture from circumcised male infants. Am J Dis Child. 1993 Sep;147(9):969-70.

10 Ramage IJ, Chapman JP, Hollman AS, Elabassi M, McColl JH, Beattie TJ. Accuracy of clean-catch urine collection in infancy. J Pediatr. 1999 Dec;135(6):765-7.

11 Herreros Fernández ML, González Merino N, Tagarro García A, Pérez Seoane B, de la Serna Martínez M, Contreras Abad MT, et al. A new technique for fast and safe collection of urine in newborns. Arch Dis Child. 2013 Jan;98(1): 27-9.

12 Altuntas N, Tayfur AC, Kocak M, Razi HC, Akkurt S. Midstream clean-catch urine collection in newborns: a randomized controlled study. Eur J Pediatr. 2015 May;174(5):57782.

13 Karacan C, Erkek N, Senel S, Akin Gunduz S, Catli G, Tavil B. Evaluation of urine collection methods for the diagnosis of urinary tract infection in children. Med Princ Pract. 2010; 19(3):188-91.

14 Herreros ML, Tagarro A, García-Pose A, Sánchez A, Cañete A, Gili P. Accuracy of a new clean-catch technique for diagnosis of urinary tract infection in infants younger than 90 days of age. Paediatr Child Health. 2015 Aug-Sep; 20(6):e30-2.
15 Kim GA, Koo JW. Validity of bag urine culture for predicting urinary tract infections in febrile infants: a paired comparison of urine collection methods. Korean J Pediatr. 2015 May;58(5):183-9.

16 Aronson AS, Gustafson B, Svenningsen NW. Combined suprapubic aspiration and cleanvoided urine examination in infants and children. Acta Paediatr Scand. 1973 Jul;62(4): 396-400.

17 Kaufman J, Fitzpatrick P, Tosif S, Hopper SM, Donath SM, Bryant PA, et al. Faster clean catch urine collection (Quick-Wee method) from infants: randomised controlled trial. BMJ. 2017 Apr;357:j1341.

18 Alam MT, Coulter JB, Pacheco J, Correia JB, Ribeiro MG, Coelho MF, et al. Comparison of urine contamination rates using three different methods of collection: clean-catch, cotton wool pad and urine bag. Ann Trop Paediatr. 2005 Mar;25(1):29-34

19 Ballouhey Q, Fourcade L, Couve-Deacon E, Cros J, Lescure V, Bahans C, et al. Urine contamination in nontoilet-trained and uncircumcised boys. Urology. 2016 Sep;95:171-4.

20 Ho IV, Lee CH, Fry M. A prospective comparative pilot study comparing the urine collection pad with clean catch urine technique in non-toilet-trained children. Int Emerg Nurs. 2014 Apr;22(2):94-7.
21 Labrosse M, Levy A, Autmizguine J, Gravel J. Evaluation of a new strategy for clean-catch urine in infants. Pediatrics. 2016 Sep; 138(3):e20160573.

22 Vaillancourt S, McGillivray D, Zhang X, Kramer MS. To clean or not to clean: effect on contamination rates in midstream urine collections in toilet-trained children. Pediatrics. 2007 Jun;119(6):e1288-93.

23 Foglia EE, Lorch SA. Clinical predictors of urinary tract infection in the neonatal intensive care unit. J Neonatal Perinatal Med. 2012 Oct;5(4):327-33.

24 Hoberman A, Wald ER, Reynolds EA, Penchansky L, Charron M. Pyuria and bacteriuria in urine specimens obtained by catheter from young children with fever. J Pediatr. 1994 Apr;124(4):513-9.

25 Mori R, Yonemoto N, Fitzgerald A, Tullus K, Verrier-Jones K, Lakhanpaul M. Diagnostic performance of urine dipstick testing in children with suspected UTI: a systematic review of relationship with age and comparison with microscopy. Acta Paediatr. 2010 Apr;99(4): 581-4.

26 Magín EC, García-García JJ, Sert SZ, Giralt AG, Cubells CL. Efficacy of short-term intravenous antibiotic in neonates with urinary tract infection. Pediatr Emerg Care. 2007 Feb; 23(2):83-6 